\title{
Blood Group Changed in a Patient with Acute Myelocytic Leukemia
}

\author{
SHAH MD. SARWER JAHAN, ${ }^{1}$ A.K.M KAMRUZZAMAN, ${ }^{2}$ MD.ISMAIL HOSSAIN, ${ }^{3}$ MD.ABDUL MATIN, ${ }^{4}$ \\ MD. ZAKIR HOSSAIN ${ }^{5}$
}

\begin{abstract}
Gain of red blood cell antigens has been described in patients with acute myelocytic leukemia (AML). This paper describes the gain of blood group A antigen in a patient with AML. At the time of diagnosis the patient's red cells showed the $0 R h(D)$ - phenotype. After induction of remission with one courses of Daunorubicin, Cytarabine, her blood group was changed to $A R h(D)$ - phenotype.
\end{abstract}

Keywords: Blood group - Acute leukemia

\section{Introduction}

Red blood cell (RBC) antigens represent inherited traits and as such, their expression should be constant throughout the life of an individual. We describe a patient in whom the expression of A antigens due to the development of Acute myelocytic leukemia $(\mathrm{AML})^{1}$. Gain of red cell antigens has been described in association with neoplastic disorders of hemopoiesis. These modification of blood group antigens may revert to normal after induction of complete remission. Significantly lower scores of A, B and $\mathrm{H}$ antigens in patients with myelodysplastic syndrome, leukemia or lymphoma has been observed. ${ }^{2}$ We report gain of blood group A antigen in patient with AML.

\section{Case Report}

Angu Roi 16 years of age daughter of Khirod healing from Parbotipur, Dinajpur was reasonably well six month back. First she develops fever which was high grade continuous in nature not associated with chills and rigor. She also complains generalized weakness in associated with fever. After 10 days of onset of fever she develops spontaneous bleeding from gum and nose. Then she was admitted in hospital.At that time her investigation profile as follows $\mathrm{Hb}-$ $4.00 \mathrm{gm} / \mathrm{dl}$, ESR-105 mm of $\mathrm{Hg}$ in first hour, Total count of

1. Assistant Professor, Department of Medicine, Rangpur Medical College \& Hospital.

2. Assistant Professor, Department of Hematology, Rangpur Medical College \& Hospital.

3. Registrar, Department of Medicine, Rangpur Medical College \& Hospital.

4. Assistant Registrar, Department of Hepatology, Rangpur Medical College \& Hospital.

5. Professor and Head, Department of Medicine, Rangpur Medical College \& Hospital.

Correspondence: Dr. Shah Md. Sarwer Jahan, Assistant Professor, Department of Medicine, Rangpur Medical College \& Hospital, Rangpur, Bangladesh.
WBC-65000/cumm, Platelet count-120,000/cumm, In Differential count nutrophil-25\%, lymphocyte$45 \%$,monocyte- $04 \%$, esonophil- $01 \%$,blast cell- $25 \%$, PCV$13.7 \%$. Urine R/E Shows-Gross haematuria. PBF suggestive of Acute Leukaemia.The bone marrow examination shows AML.At that time her blood group was $\mathrm{O}(-)$ ve. Then six unit $\mathrm{O}(-)$ ve blood transfusion given and discharged with Tab.Marcaptopurine (50mg) $12 \mathrm{hrly}$ and Tab.Prednisolone $(60 \mathrm{mg})$ daily and advised to repeat $\mathrm{CBC}$ with Urine $\mathrm{R} / \mathrm{E}$ after 6 weeks. After that she admitted in RpMC\&H on 02.06.2012 with the complain of haematuria.On admission her $\mathrm{Hb}-6.5 \mathrm{gm} /$ $\mathrm{dl}$, total count of WBC-70000/cumm, differential count was nutrophil-05\%,lymphocyte-15\%,Monocyte-00\%,Esonophil$00 \%$,blast cell- $80 \%$ So we advised her for blood transfusions and five unit of $\mathrm{O}(-)$ ve blood was given. Then we go for remission induction by daunorubicin and cytarabine. One weeks after chemotherapy she develop menorrhogia and at that time her total platelet count was $8000 /$ cumm. So we decide to give fresh blood but surprisingly her blood group shows A(-)ve. Then we repeat the test in five different labs. All lab reports shows $\mathrm{A}(-)$ ve blood, we then consult we blood transfusion department about this problems and they confirms us that at present her blood group is $\mathrm{A}(-)$ ve.Then two unit of A (-)ve fresh human blood was given without any hazards.

\section{Discussion}

Either loss or appearances of RBC antigens have been reported to occur in a number of malignancies (both hematologic and solid). The first report, and those which make up the majority of reports, ${ }^{3-6}$ have involved alterations in the expression of ABO blood group antigens. In addition to the $\mathrm{ABO}$ blood group system, multiple other antigens and antigen systems/collections have been reported to be affected by malignancy. These changes in blood group antigens can result from a number of mechanisms; the 
mechanism involved depends on the antigen involved as well as on the disease. For hematopoietic diseases, the loss of expression predominantly results from a mutation within a stem cell that affects antigen production. If the affected clone gives rise to a significant proportion of the RBCs, it may result in the appearance of a mixed population of antigennegative and antigen-positive RBCs. If the clone has given rise to all or the majority of the RBCs present, then complete loss of the antigen occurs.

Loss or appearance of ABO antigens presents as a discrepancy in the forward and reverse typing of patients. Because $\mathrm{ABO}$ typing is routinely performed in pre transfusion testing, $\mathrm{ABO}$ antigens are the most frequently reported blood group antigen change identified in malignancy. In order to detect changes in other systems or antigens, one must either have known the patient's previous phenotype or observed a change or detect a return to their original phenotype during the course of treatment. The production of ABO antigens results from the interactions of two glycosyl transferases. The first enzyme, $H$ transferase, adds a fructose to the terminal galactose of precursor substance. The $\mathrm{H}$ substance generated is then acted on by the $A$ and/or $B$ transferases that add an $\mathrm{N}$-acetylgalactosamine or a galactose, respectively. In leukemias and other hematopoietic disorders, two possible mechanisms exist to explain the weakening of ABO antigens. In the first, the $A$ and/or $B$ transferase are inactivated. The result is weakening or loss of the A and/or $\mathrm{B}$ antigens with a concurrent increase in $\mathrm{H}$ antigen because $\mathrm{H}$ antigen is no longer converted to $\mathrm{A}$ and $\mathrm{B}$ antigen, a finding that has seen by a number of authors. ${ }^{5,7-10}$ It has been suggested that, at least in CML, such a change may result from inactivation of the $A$ and/or $B$ transferase genes on chromosome 9 through the generation of the $9 ; 22$ chromosomal translocation(Philadelphia chromosome). ${ }^{(11)}$ The gene encoding the $A$ and $B$ transferases is located at $9 \mathrm{q} 34$, the area in which the break point for the chromosomal translocation occurs. As a result, the translocation could disrupt the gene, thereby preventing expression of the transferase. Although this is a possibility, ABO changes are more commonly seen in acute myelogenous leukemia (AML), where this chromosomal translocation is uncommon. ${ }^{9}$ One study of 12 patients with acute myelogenous leukemia (AML) and weakening of the $\mathrm{ABO}$ antigens suggested that the $A B O$ gene inactivation was not random. This suggested the presence of genomic imprinting, defined as the differential expression of a gene as determined by whether it is inherited from the mother or father, in determining which gene was inactivated. The authors of this report realized that additional study was needed, given the small number of patients' examined. ${ }^{12}$ The second possible mechanism for the gain/ loss of ABO antigens involves the inactivation of the $H$ transferase encoded at 19q13. The loss of $H$ transferase would result in decreased $\mathrm{H}$ substance and a resulting decrease in $A$ and/or $B$ substance, again identified in a number of patients. ${ }^{7,9-13}$ Loss of $\mathrm{ABO}$ antigens is of more than academic interest. In a number of cases, the loss or weakening of $\mathrm{ABO}$ antigens has been detected prior to the diagnosis of the underlying hematopoietic malignancy, frequently in the setting of long-standing myelodysplasia. ${ }^{10,14,15}$ It has been suggested that the identification of a loss/gain of ABO antigens should result in the search for an underlying hematopoietic malignancy. Finally, changes in ABO antigens have also mirrored the course of the malignancy with return of the original blood type upon remission and reappearance of antigen-negative cells with recurrence. ${ }^{6-16}$ In the case of solid tumors such as pancreatic, gastric, colonic, ovarian, and biliary carcinomas, an apparent loss of ABO antigens also can be seen. ${ }^{16}$ The term "apparent" is used because unlike in hematopoietic malignancies, the number of $A, B$, and $H$ antigens on the RBCs are not altered. Instead, the tumors secrete large amounts of soluble $A$ and/or $B$ substance. The soluble blood group substance that neutralizes the typing reagents resulting in the apparent loss of $\mathrm{A}$ and $\mathrm{B}$ antigens can be overcome by washing the RBCs thoroughly prior to forward typing in order to remove the plasma with its soluble blood group substance. ${ }^{17}$ This finding is usually detected as a discrepancy between the forward and reverse typing of the patient.

\section{Conclusion}

Changes in RBC antigen phenotype rarely occur. They are most frequently seen in association with hematologic malignancies but can be seen in association with solid tumors as well. The identification of these changes represents more than just an academic exercise. Changes have been identified prior to the diagnosis of the responsible underlying malignancy and have heralded relapse of the malignancy. In addition, the realization that $\mathrm{ABO}$ antigen had been gain in our patient. For these reasons, it is important for blood bank professionals to be aware of the existence of this phenomenon.

\section{Conflict of Interest : None}

\section{References}

1. Blood group change in a patient with blastic transformation of a myeloblastic syndrome. N. Xiros, H. Northoff, B. Anger, W. Heit and H. Heimpel Annals OF Hematology vol 54,Num 5(1987), 275-280.

2. Red blood cell antigen changes in malignancy: case report and review. J.L. Winters and D.S. Howard, Immunohematology, Vol 17, Num 1, 2001. 
3. Kolins J, Holland PV, McGinniss MH. Multiple red cellantigen loss in acute granulocytic leukemia. Cancer 1978; 42: $2248-53$

4. Satheesh P, Thomas M, Rajan GB. Change in the ABO blood group phenotype. J Assoc Physicians India 1994; 42: 830 .

5. Chiewsilp P, Atichartakarn V. Change of red cell B antigen in a leukemic patient. J Med Assoc Thailand 1981; 64: 413-7.

6. Yamada K, Yoshizaki Y, Fujii Y, et al. Concurrent ABO blood type change and erythrophagocytosis by leukemic cells in a case of acute myeloblastic leukemia. Acta Haematol Jpn 1985; 48:1409-13.

7. van der Hart M, van der Veer M, van Loghem JJ.Change of blood group B in a case of leukemia. Vox Sang 1962; 7: 449-53.

8. Hoogstraten B, Rosenfield RE, Wasserman LR.Change of ABO blood type in a patient with leukemia. Transfusion 1961; 1: 32-5.

9. Crookston MC. Anomalous ABO, H, Ii phenotypes in disease. In: Garratty G, ed. Blood group antigens and disease. Arlington, VA: American Association of Blood Banks, 1983: $67-84$.

10. Yoshida A, Kumazaki T, Dave V, Blank J, Dzik WH. Suppressed expression of blood group B antigen and blood group galactosyltransferase in a preleukemic subject. Blood 1985; 66: 990-2.

11. Reid ME, Bird GW. Associations between human red cell blood group antigens and disease. Transfus Med Rev 1990; 4: 47-55.

12. Dobrovic A, O'Keefe D, Sage RE, Batchelder E. Imprinting and loss of ABO antigens in leukemia. Blood 1993; 82: 1684-5.

13. Kuhns WJ, Oliver RT, Watkins WM, Greenwell P.Leukemiainduced alterations of serum glycosyltransferase enzymes. Cancer Res 1980; 40: 268-75.

14. Salmon C. Blood group changes in preleukemic states. Blood Cells 1976;2:211-20.

15. Lopez M, Bonnet-Gajdos M, Reviron M, Janvier D, Huet M, Salmon C. An acute leukaemia augured before clinical signs by blood group antigen abnormalities and low levels of $\mathrm{A}$ and $\mathrm{H}$ blood group transferase activities in erythrocytes. Br J Haematol 1986; 63: 535-9.

16. Gold ER, Tovey GH, Benny WE, Lewis FJW. Changes in group A antigen in a case of leukaemia. Nature 1959; 183: 892-3.

17. Treacy M, Geiger J, Goss MF. Substances in serum causing interference with blood group determination. Transfusion 1967; 7: 443-6. 